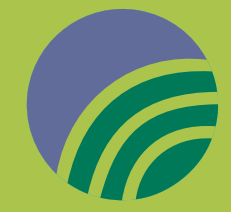

\title{
2019 MRS Spring Meeting offers something for everyone
}

mrs.org/spring2019

$\mathrm{T}$ he Materials Research Society (MRS) held its Spring Meeting in Phoenix, Ariz., from April 22-26, attracting an interdisciplinary and international audience. The Meeting Chairs, Yuping Bao (The University of Alabama), Bruce Dunn (University of California, Los Angeles), Subodh Mhaisalkar (Nanyang Technological University), Ruth Schwaiger (Karlsruhe Institute of Technology-Institute for Applied Materials), and Subhash L. Shinde (University of Notre Dame), organized 60 symposia, grouped into seven topical clusters, which comprised the technical core of the meeting: General Interest; Broader Impact; Characterization, Processing and Theory; Electronics and Photonics; Energy and Sustainability; Quantum and Nanomaterials; and Soft Materials and Biomaterials.

\section{MRS Awards}

MRS presented several awards: Stig Helveg (Haldor Topsoe A/S) received the Innovation in Materials Characterization Award; Hongyou Fan (Sandia
National Laboratories and The University of New Mexico) received the Mid-Career Researcher Award; Sheng Xu (University of California, San Diego) received the Outstanding Young Investigator Award; Meyya Meyyappan (NASA Ames Research Center) received the MRS Impact Award; Kaifu Bian (Sandia National Laboratories) and Nicholas E. Jackson (Argonne National Laboratory) received MRS Postdoctoral Awards; and Huachen Cui, Ryan Hensleigh, Hongshun Chen, and Xiaoyu Zheng (Virginia Tech) won

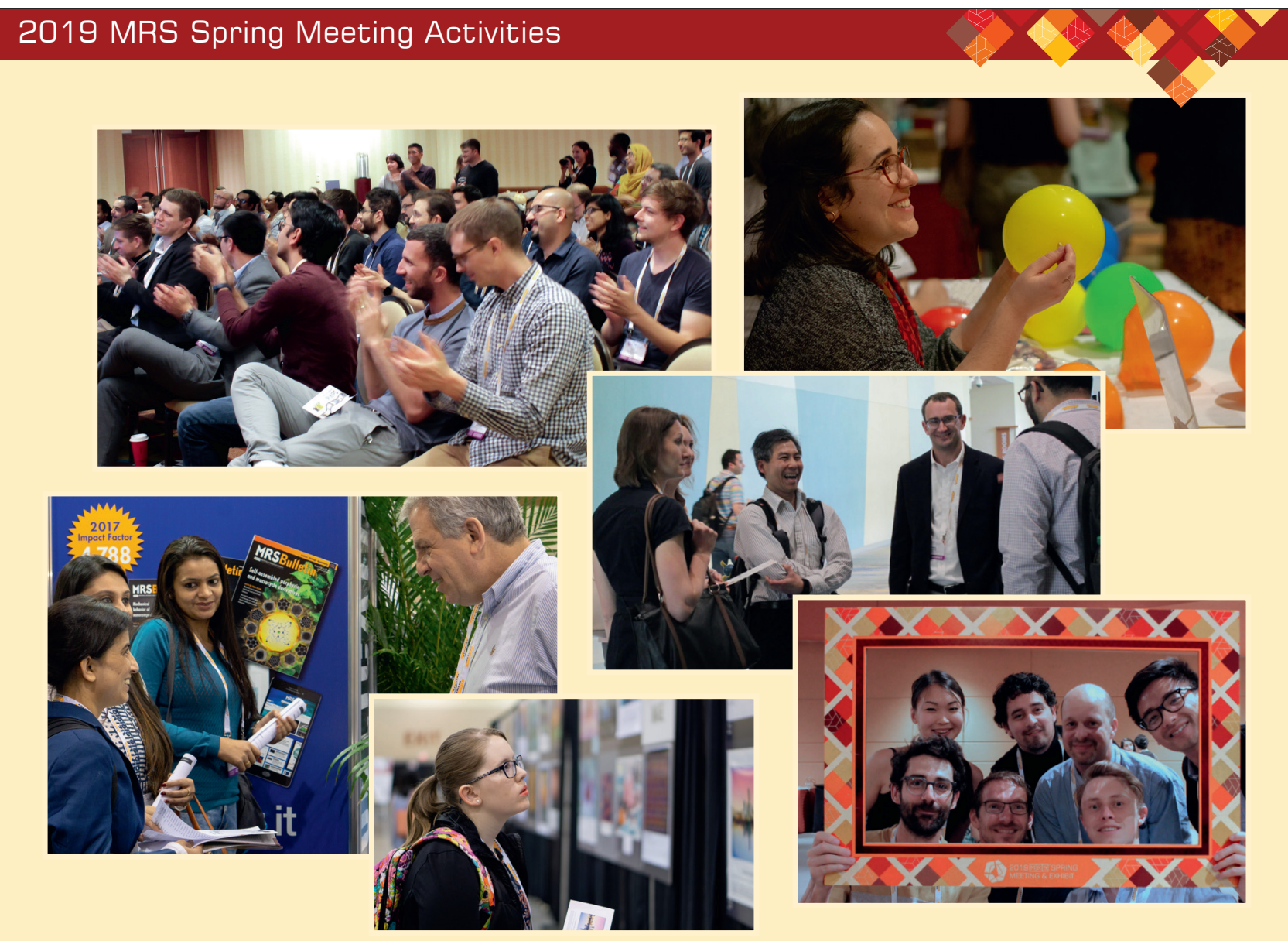

(Left to right) Audience at the inaugural PowerPoint Karaoke; demonstration at the Student Mixer; engaged attendees discussing publication opportunities; networking during coffee breaks; Science as Art voting; and fun photo opportunities at the Publications Reception. 
the 2018 Journal of Materials Research Paper of the Year Award for their article, "Additive Manufacturing and size-dependent mechanical properties of three-dimensional microarchitected, high-temperature ceramic metamaterials." Helena Van Swygenhoven-Moens of the Paul Scherrer Institute and École Polytechnique Fédérale de Lausanne, delivered the plenary talk, "Synchrotron Light to Investigate Materials In Operando," as the recipient of The Fred Kavli Distinguished Lectureship in Materials Science. Timothy J. Bunning, Air Force Research Laboratory, was selected for the 2019 MRS Communications Lecture and presented "Dynamic Optical Properties of Gold Nanoparticles/Cholesteric LiquidCrystal Arrays."

The MRS Awards Program strives to acknowledge outstanding contributors to the progress of materials research and to recognize their accomplishments. Nominate a colleague for one of the many MRS Awards to be presented at the 2020 MRS Spring Meeting by August 1. For addtional information, visit mrs.org/awards.

\section{Symposium X-}

\section{Frontiers of Materials Research}

Four Symposium X presentations were featured at the 2019 MRS Spring Meeting. Molly M. Stevens, Imperial College London, discussed "Designing Bio-Responsive Hybrid Materials," in which she described a wide range of examples from her group that employs materials in biomedical applications, as well as applies advanced materials characterization techniques to understand and improve the materials.

In her presentation on "Hydrogen and Fuel-Cell Technology Perspectives," Sunita Satyapal, US Department of Energy (DOE), focused on the progress in and status of hydrogen and fuel-cell technologies, the DOE's H2@Scale initiative, R\&D needs and challenges, and how materials science and research collaborations could help address those.

Jonathan Arenberg, Northrop Grumman Aerospace Systems, discussed "NASA's James Webb Space Telescope" and described the mission, design challenges, and development of NASA's next

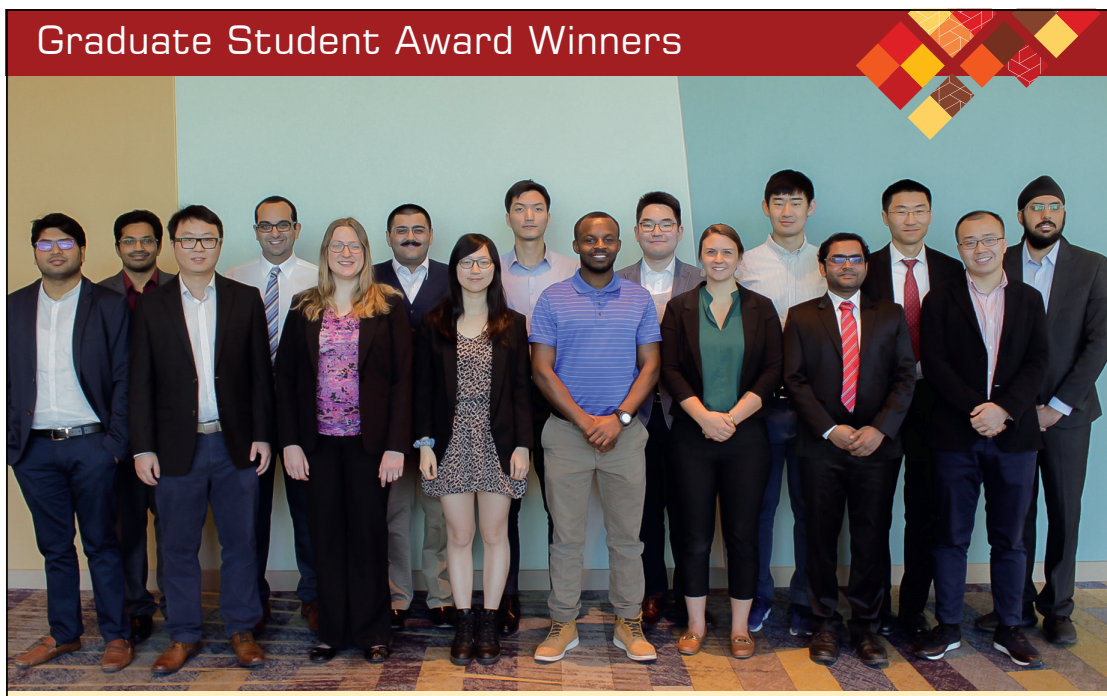

(Left to right) Subhajit Roychowdhury, Jawaharlal Nehru Centre for Advanced Scientific Research; Rohit John, Nanyang Technological University; Yixiu Wang, Purdue University; Peter Attia, Stanford University; Rainie Nelson, lowa State University of Science and Technology; Aashutosh Mistry, Purdue University; Wen-Hui Cheng, California Institute of Technology; Hyunjoong Chung, University of Illinois at Urbana-Champaign; Aristide Gumyusenge, Purdue University (Arthur Nowick Graduate Student Award recipient); Hyunwoo Yuk, Massachusetts Institute of Technology; Jennifer Boothby, The University of Texas at Dallas; Xiaoxing Xia, California Institute of Technology; Amitava Banerjee, Uppsala University; Andrew Meng, Stanford University; Lichen Liu, Universitat Politècnica de València; Arashdeep Thind, Washington University in St. Louis. Missing from photo: Zhaoqianqi Feng, Brandeis University; Joon Sang Kang, University of California, Los Angeles.

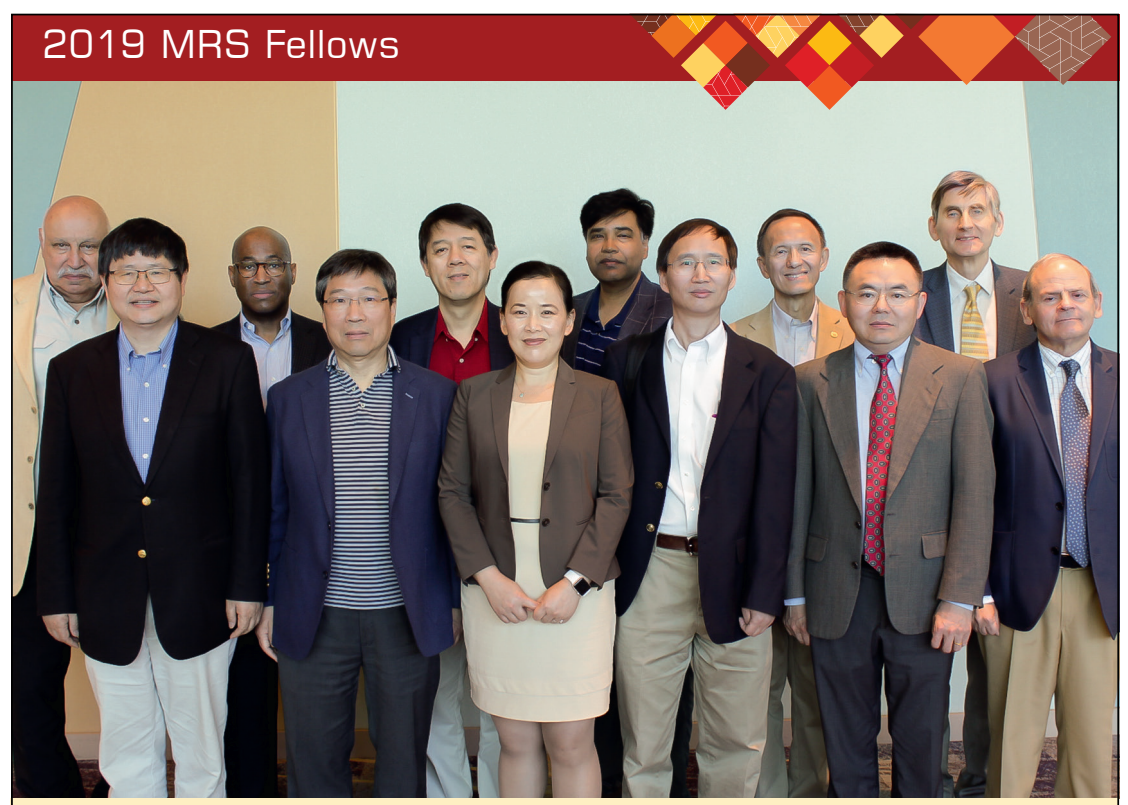

(Left to right) Miguel José Yacaman, The University of Texas at San Antonio; Sheng Dai, Oak Ridge National Laboratory and The University of Tennessee, Knoxville; Peter F. Green, National Renewable Energy Laboratory; Yue Kuo, Texas A\&M University; Xiao Cheng Zeng, University of Nebraska-Lincoln; Haiyan Wang, Purdue University; Sudipta Seal, University of Central Florida; Yuntian Zhu, North Carolina State University; Yimei Zhu, Brookhaven National Laboratory; Ji-Cheng (JC) Zhao, The Ohio State University; Jesús A. del Alamo, Massachusetts Institute of Technology; Javier Llorca, IMDEA Materials Institute and Universidad Politécnica de Madrid. Missing from photo: Michael Chabinyc, University of California, Santa Barbara; Mary E. Galvin, University of Notre Dame; Steven G. Louie, University of California, Berkeley; Natalie StingelinStutzmann, Georgia Institute of Technology; Paul S. Weiss, University of California, Los Angeles; Matthias Wuttig, RWTH Aachen University. 
leap in space science. He explored how the design responds to mission requirements and produces the performance necessary to achieve the mission's goals.

Bart Biebuyck, Fuel Cells and Hydrogen Joint Undertaking (FCH JU), gave an overview of the progress on fuel cell and hydrogen technologies in Europe in his talk, "Development of Fuel Cells and Hydrogen Technologies in Europe Toward Commercialization from 2020 Onward." The FCH JU supports stretches from research and demonstration projects in the different areas of $\mathrm{FCH}$, including energy and transport applications.

\section{Meeting features}

MRS hosted the MRS Frontiers Reception: Building Communities, to engage materials researchers with shared interests related to quantum, bio, sustainability, artificial intelligence, and other topics. Ideas emerging from this engagement can feed future programming, publications, and outreach activities.

\section{Science as Art}
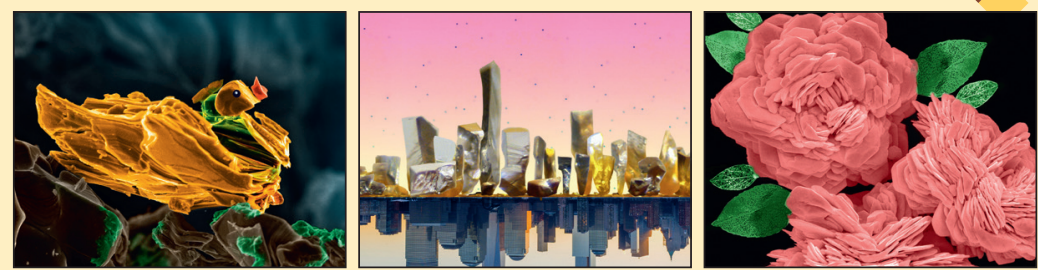

Congratulations to the Science as Art competition first-place winners: Kanit Hantanasirisakul, Drexel University, for "Nanoduck"; Duhan Zhang, Cornell University, for "Manhattan Skyline at Twilight"; and Han Zhang, The Chinese University of Hong Kong, for "Gold Peony."

Through interactions across these diverse interest groups in a variety of planned engaging and interactive ways, participants were able to build and seek the interrelationship between shared communities and secure collaborations for new research initiatives and grant applications.

The Open Data Challenge started as a way for people to contribute to the materials science and informatics communities by curating or developing a materials data set and applying materials informatics techniques to data sets to learn something new. In the end, the participants needed to build a presentation to share their methodology and results with the community.

The kick-off webinar occurred in midDecember 2018, with Josh Tappan and Malcolm Davidson (Citrine Informatics) leading the charge and with support through Citrine and MRS. Winners were announced at the 2019 MRS Spring Meeting in Phoenix. First-place winners were Peter Attia and Norman Jin from Stanford University

\section{Tharnk you! Spring Meeting Symposium Support}

1-Material Inc

3M

Accurion Inc.

ACS Energy Letters | ACS Publications

Advanced Materials Technologies | Wiley

Applied Materials, Inc.

Applied Physics Letters | AIP Publishing

Arizona State University

Army Research Office

ASM International

ASU LightWorks

attocube systems AG

AVANCIS GmbH

Bio-Logic USA

Bosch

Bruker

CBMM North America, Inc.

CEA, LETI

\section{CEM Corporation}

Center for Integrated Nanotechnologies, Los Alamos National Laboratory

Chemical Science |

Royal Society of Chemistry

Codex International

College of Engineering, Penn State
CreaTec GmbH, represented by Sentys Inc.

U.S. Department of Energy

Direct Electron

EMD Performance Materials (a business of Merck KGaA, Darmstadt, Germany)

Energy \& Environmental Science

Royal Society of Chemistry

Enli Technology Co., Ltd.

Entegris, Inc.

Environmental Science \& Technology| ACS Publications

Environmental Science \& Technology Letters | ACS Publications

First Solar, Inc.

Gatan, Inc.

Henan University

Hitachi High Technologies America, Inc

IFP Energies nouvelles

InnovationLab GmbH

IOP Publishing

Joule $\mid$ Cell Press

Journal of Materials Chemistry A

Royal Society of Chemistry

JPhys Energy | IOP Publishing

JPhys Materials | IOP Publishing
Los Alamos National Laboratory

Materials Horizons

Royal Society of Chemistry

Matter| Cell Press

Media Tech. Co., Ltd.

Michael Saliba

Microsoft Quantum

MilliporeSigma

Murata Electronics North America, Inc.

Nanoscale Advances \& Chemical

Science | Royal Society of Chemistry

National Renewable Energy Laboratory

National Science Foundation

Netherlands Society for Biomaterials and Tissue Engineering

NeuroLux, Inc.

Neware Technology (Hong Kong) Ltd. Office of Naval Research

Oxford Instruments NanoScience

Pacific Northwest National Laboratory

Pascal Co., Ltd.

Pine Research Instrumentation

Prevac

Protochips

Radiant Technologies

Rocky Mountain Vacuum Tech, Inc.
RSC Advances | Royal Society of Chemistry

Rutgers University - Newark Chancellor's Office

Rutgers University - Newark Department of Chemistry

Sandia National Laboratories

Scienta Omicron, Inc.

Scribner Associates, Inc.

Solar RRL | Wiley

SPI Supplies, Division of Structure Probe, Inc.

Sustainable Energy \& Fuels |

Royal Society of Chemistry

TEL

The Advanced Photon Source (APS) at Argonne National Laboratory

The Southern Indiana Section of the American Chemical Society (SISACS)

Thermo Fisher Scientific

University at Buffalo, Center of Excellence in Materials Informatics

University of Luxembourg / Fonds national de la recherche (Luxembourg) University of Michigan Energy Institute Vigor Tech USA, Ltd.

WITec Instruments Corp.

Xiamen Xinji Technology Ltd 
("Battery Cycling During Extreme Fast Charging"); second-place winners were Viraj Shah, Balaji Sesha Sarath Pokuri, Rahul Singh, Ameya Joshi, Chinmay Hegde, Baskar Ganapathysubramanian, and Soumik Sarkar from Iowa State University of Science and Technology ("Deep Generative Models for Creating Synthetic Microstructures"); and thirdplace winners were Zhe Shi and Wujie Wang from the Massachusetts Institute of Technology ("Deep Spatial Fingerprint Neural Network Potential Development").

Participants were judged on the clarity of their communication, novelty of their data set and analysis approach, and explanation of their methodology. More information can be found at mrs.org/ open-data-challenge.

PowerPoint Karaoke was held for the first time as a challenge to both the author of a slide and the presenter to improve their communication skills while under a strict time limit. After the event, there was time to chat, laugh, snack, and network with other participants in a relaxed environment.

The participants had three minutes to present a slide. The challenge of the event was to get the best information out of the slide. A jury announced the winning presenter according to the best performance and author for the clearest slide.

The professional development workshop on Designing Sustainability into Materials Research focused on aspects that limit sustainable materials development, including materials availability, reuse, recycling, and recovery. Additional aspects include environmental and human health effects, such as carcinogenicity and acute water toxicity, which can be used to screen for safer materials selection choices in research activities and in manufacturing and production.

The workshop also entailed various modes of learning, including presentations, videos, team discussions, and direct data access to information sources that can facilitate sustainable materials development in research activities.

Olivia A. Graeve, University of California, San Diego, was the presenter at the MRS Women in Materials Science \& Engineering Breakfast on Wednesday. These gatherings feature a timely discussion
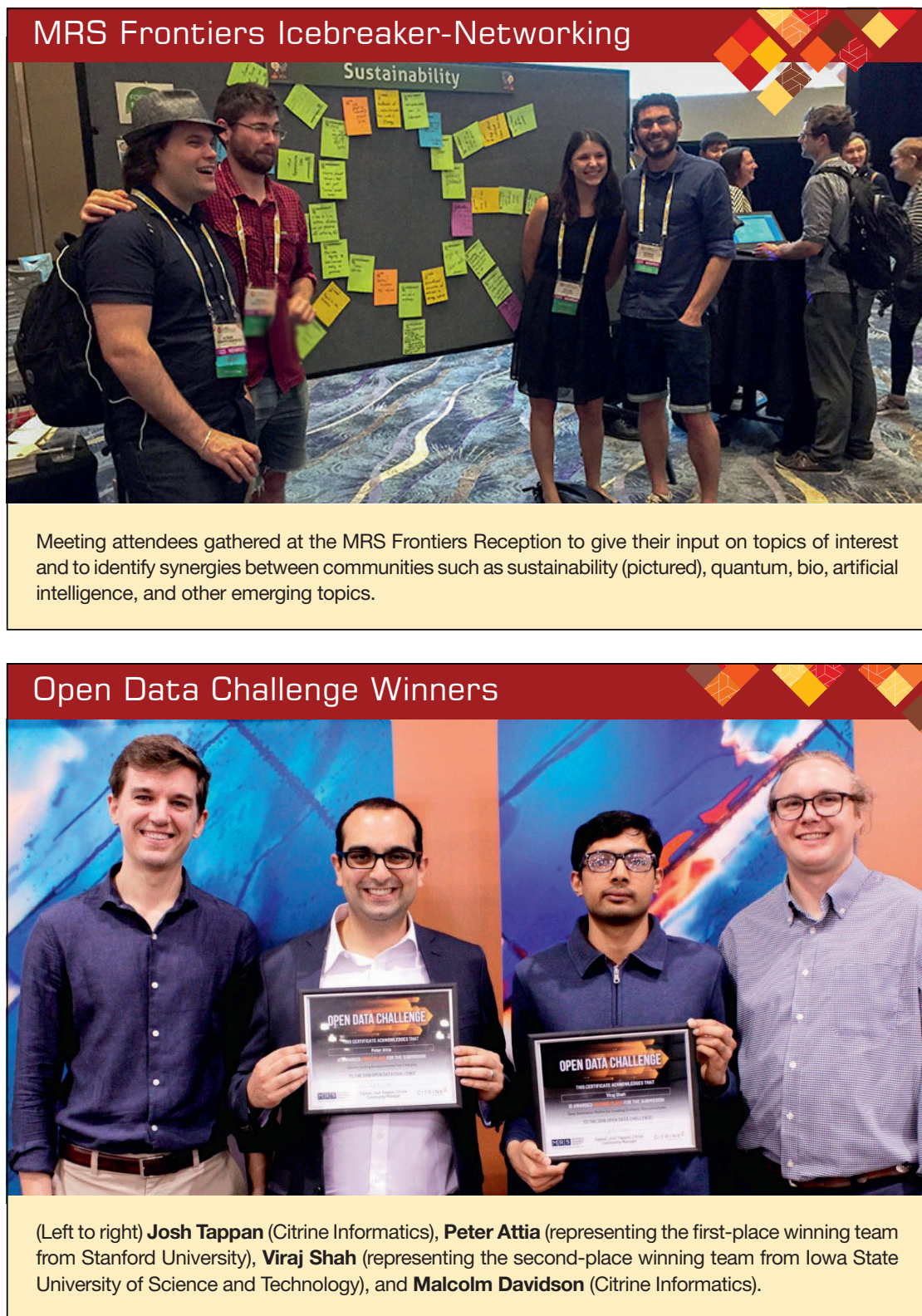

of issues facing women in the materials science and engineering profession, and provide an opportunity for networking for women in MS\&E from around the world.

Graeve's discussion featured a personal narrative about her journey through diversity and equity in sciences and engineering as a Latino woman. She also shared views on multiculturalism, empathy, and the building of human connections. "As a child, you are told, 'this is the status quo.' When you grow up with segregation around you, how do you change your mind? When does the switch happen?"

Graeve has been involved in many activities related to the recruitment and retention of women and Hispanic students and has received several awards. Forbes Magazine named her one of the 100 Most Powerful Women of Mexico (2017).

MRS recognizes the critical role volunteers play in the Society. The growth and success of MRS is due to the energy, enthusiasm, and vision of many member volunteers, to whom we are most grateful.

Many presentations from the 2019 MRS Spring Meeting (mrs.org/ spring2019) are available through news coverage on MRS TV (mrs.org/mrs-tv) and the MRS Meeting Scene $e^{\circledR}$ (materials. typepad.com). 